\title{
Racemose Neurocysticercosis Masquerading High-Grade Glial Neoplasm: A Rarest Presentation of Common Disease
}

\author{
Rakesh Kumar $^{1}$ Deepak Kumar Singh ${ }^{2}$ Neha Singh ${ }^{3}$ \\ ${ }^{1}$ Department of Neurosurgery, King George's Medical University, \\ Lucknow, Uttar Pradesh, India \\ 2 Department of Neurosurgery, Dr. Ram Manohar Lohia Institute of \\ Medical Sciences, Lucknow, Uttar Pradesh, India \\ 3 Department of Radiodiagnosis, King George's Medical University, \\ Lucknow, Uttar Pradesh, India \\ Indian J Neurosurg 2016;5:117-119. \\ Address for correspondence Dr. Rakesh Kumar, 1/297, Virat Khand, \\ Gomtinagar, Lucknow, Uttar Pradesh, India \\ (e-mail: rksingh2226@gmail.com).
}

We are presenting a case of racemose neurocysticercosis presented with symptoms of increased intracranial pressure and radiologically mimicking high-grade glioma, which was managed surgically with good recovery. Neurocysticercosis is the commonest parasitic disease of the human nervous system having pleomorphic clinical manifestations, which depend on the location, number, and stage of the cysts at presentation. Atypical presentation of cerebral cysticercosis may mimic glioma and may be difficult to differentiate from neoplastic condition. Differ from the usual medical management, they may require urgent surgical intervention.

\section{Introduction}

Neurocysticercosis (NCC) is the infection of the central nervous system (CNS), which is caused by the larval form (Cysticercus cellulosae) of Taenia solium. It is the most common parasitic disease of the human nervous system. ${ }^{1}$ It can affect the parenchyma, subarachnoid space, or intraventricular system. The clinical manifestations are dependent on the location, number, and stage of the cysts at presentation. ${ }^{2}$ Single, large racemose cysticercus may be difficult to differentiate from neoplastic condition. ${ }^{3}$ Racemose NCC may present with symptoms of increased intracranial pressure and can mimic high-grade glioma radiologically. This atypical presentation of this common disease is extremely rare, and only few case reports are available in the published literature, with surgical management having good outcome.

\section{Case Report}

A 35-year-old man from Faizabad, India, presented with complain of headache for the last 2 months and two to three episodes of vomiting. He was a vegetarian, and laborer by occupation. On taking detailed history with his wife, history of change in behavior was present. On examination, the patient was conscious and oriented to time, place, and person without any focal neurological deficit. On fundus examination, bilateral papilledema was present, and rest of the neurological examination was normal.

Magnetic brain imaging (MRI) of the brain was done, which revealed a large, well-defined multilobulated heterogeneous space occupying lesion in the left frontal lobe, which was hypointense on T1-weighted images and hyperintense on T2-weighted images. Perilesional edema with mass effect and subfalcine herniation were present (-Fig. 1).

On the basis of presentation and radiological findings, high-grade glioma was diagnosed.

The patient was on intravenous antiedema, antiepileptic, and antiemetic medications, and planned for early surgery. Left frontal craniotomy with complete excision of lesion was done. In the peroperative period, there was a multilobulated, moderately firm vascular lesion, having maintained plane of received

January 5, 2016

accepted

January 14, 2016

published online

July 14, 2016
DOI http://dx.doi.org/

10.1055/s-0036-1581973. ISSN 2277-954X.
(C) 2016 Neurological Surgeons' Society of India

License terms

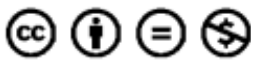




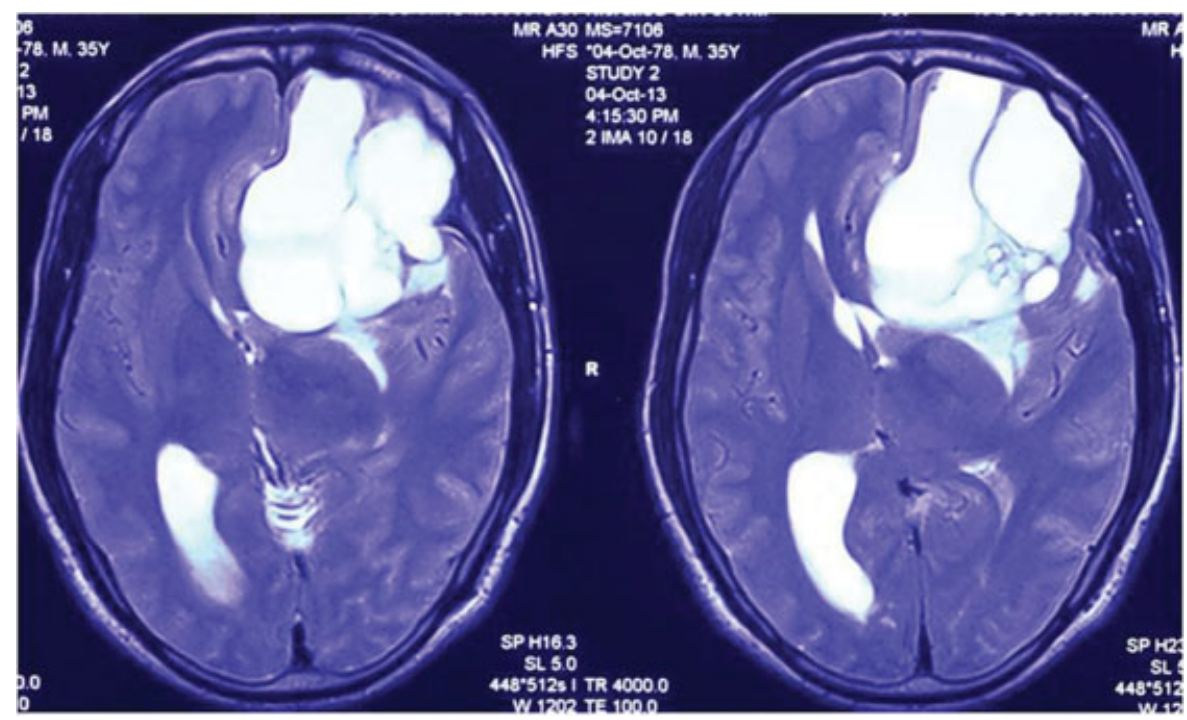

Fig. 1 MRI brain showing a large multilobulated, heterogeneous, space-occupying lesion in the left frontal lobe with perilesional edema, mass effect, and subfalcine herniation.

dissection with brain parenchyma. On histopathology, racemose NCC was confirmed (-Fig. $\mathbf{2 A}$, B). In the postoperative period, intravenous antiedema and antiepileptic medication was continued. After histological confirmation, oral albendazole (15 $\mathrm{mg} / \mathrm{kg} /$ day) along with oral steroid was started.

Postoperatively, the patient recovered well and was discharged on day 7. On the last follow-up after 6 months, the patient is symptom-free and doing well without any neurological deficit.

\section{Discussion}

NCC is the infection of the CNS by the larval form ( $C$. cellulosae) of T. solium. ${ }^{1}$ Humans and pigs are the definitive and intermediate host, respectively. ${ }^{1}$ There are four stages of development of a parenchymal larval cyst: vesicular, colloidal, nodular, and calcified. Vesicular cyst is viable with scolex existing with cyst as eccentric nodule without enhancement due to minimal host response. The colloid cyst has viscous and turbid fluid without well-defined scolex.

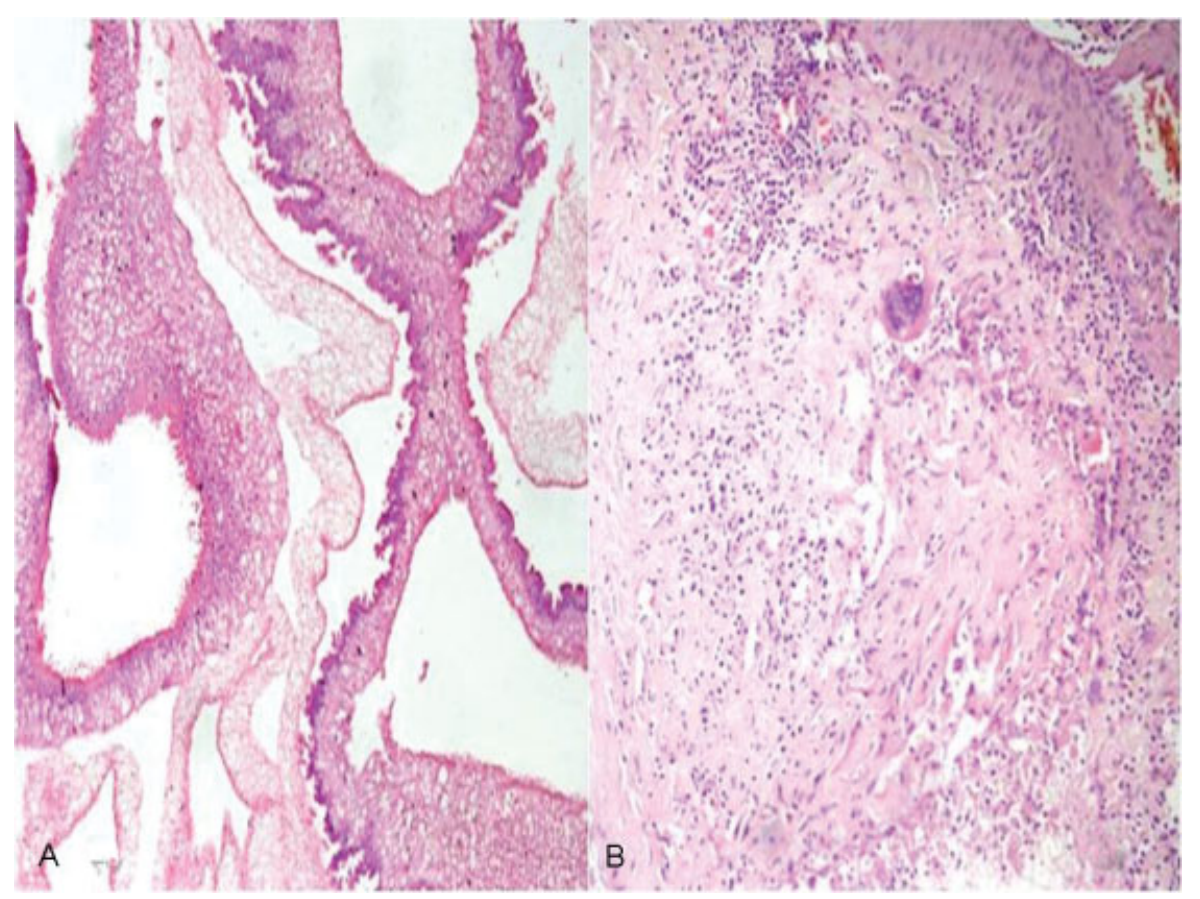

Fig. 2 (A) On H\&E $\times 40$ and on (B) H\&E $\times 10$ bladder wall of parasite comprising an outer eosinophilic cuticular layer and subcuticular layer with dark staining nuclei consistent with neurocysticercosis. 
After the migration of fluid from daughter cyst, there is a strong immune response in the surrounding parenchyma, which strongly enhances on contrast in computed tomography (CT) scan and MRI. Nodular cyst is formed after degeneration of cyst and having some contrast enhancement. Finally, cyst gets calcified and appears as punctuate calcification on CT scan. The cyst may continue to grow and also may be difficult to differentiate from neoplastic lesion. The clinical manifestations are dependent on the location, number, and stage of the cysts at presentation. ${ }^{2}$ Seizures are the commonest manifestation occurring in 50 to $80 \%$ of patients. The other clinical manifestations may be observed in the form of raised intracranial pressure, focal deficit, signs of meningitis, mental changes, myeloradiculopathy, and endocrinopathy. ${ }^{4}$ The diagnosis of NCC is difficult because of nonspecific clinical manifestations, nonpathognomonic neuroimaging, and low sensitivity and specificity of serological tests. A revised diagnostic criterion based on objective clinical, imaging, immunological, and epidemiological data was proposed by Del Brutto et al in 2001, which has two degrees of diagnostic certainty, either definitive or probable diagnosis. ${ }^{5}$ Neuroimaging is the mainstay of diagnosis of NCC. NCCT scan having sensitivity and specificity of over $95 \%$ in the diagnosis of NCC, is usually the first investigation. Rajshekhar et al described CT scan criteria for differentiating NCC and tuberculoma. ${ }^{6}$ MRI is the most accurate technique to assess degree of infection, location, and evolutionary stage of the parasite. The colloid and vesicular cyst appears hyperintense on T1-weighted MRI with contrast enhancement on gadolinium injection. ${ }^{4}$ Serological tests are of little value clinically, having sensitivity of 65 to $98 \%$ and a specificity of 67 to $100 \%$, depending on the specific test used and the cyst burden, location, and phase of infection. ${ }^{7}$

The available treatment modalities for patients with NCC are: cysticidal agents, corticosteroids (as antiinflammatory), antiepileptic drugs (to prevent or decrease the severity and number of seizures), and surgery (either for removal of the cyst or for CSF diversion in case of hydrocephalus). García et al in 2002 analyzed the current evidence and reported consensus guidelines for the management of NCC. ${ }^{8}$ Surgery is indicated for cysts exhibiting local compression of brain and cranial nerves, pseudotumor (edema) refractory to medical treatment, hydrocephalus, intraventricular cysticercosis, intracranial hypertension, spinal cysticercosis with cord/root compression, and ocular cysts. ${ }^{9}$ For parenchymatous NCC, stereotactic excisional biopsy or open craniotomy and cyst removal is recommended for a single giant cortical cyst or large clumps exhibiting tumor-like behavior on surgically accessible location. Supratentorial decompressive craniotomy, craniectomy, or lobectomy is done when there is pseudotumor-type edema, refractory to medical treatment. $^{9}$

\section{Conclusion}

The clinical manifestations of NCC are pleomorphic and may range from asymptomatic to life-threatening presentations. One of the rare forms of this common disease may present with life-threatening sign and symptoms of intracranial space-occupying lesion, difficult to differentiate from neoplastic conditions. An early diagnosis and urgent management in the form of surgical intervention in these patients result in very good outcome.

\section{References}

1 Alsina GA, Johnson JP, McBride DQ Rhoten PR, Mehringer CM, Stokes JK. Spinal neurocysticercosis. Neurosurg Focus 2002; 12(6):e8

2 Brutto Del OH, Sotelo J, Roman G. Neurocysticercosis: A Clinical Handbook. Lisse: Swets \& Zeitlinger; 1998

3 Sabel M, Neuen-Jacob E, Vogt C, Weber F. Intracerebral neurocysticercosis mimicking glioblastoma multiforme: a rare differential diagnosis in Central Europe. Neuroradiology 2001; 43(3):227-230

4 Sinha S, Sharma BS. Neurocysticercosis: a review of current status and management. J Clin Neurosci 2009;16(7):867-876

5 Del Brutto $\mathrm{OH}$, Rajshekhar V, White AC Jr, et al. Proposed diagnostic criteria for neurocysticercosis. Neurology 2001;57(2): 177-183

6 Rajshekhar V, Haran RP, Prakash S, Chandy MJ. Differentiating solitary small cysticercus granuloma and tuberculomas in patients with epilepsy. Clinical and computerized tomographic criteria. J Neurosurg 1993;78(3):402-407

7 Wilson M, Bryan RT, Fried JA, et al. Clinical evaluation of the cysticercosis enzyme-linked immunoelectrotransfer blot in patients with neurocysticercosis. J Infect Dis 1991;164(5): 1007-1009

8 García HH, Evans CA, Nash TE, et al. Current consensus guidelines for treatment of neurocysticercosis. Clin Microbiol Rev 2002; 15(4):747-756

9 Colli BO, Martelli N, Assirati Júnior JA, et al. Cysticercosis of the central nervous system. I. Surgical treatment of cerebral cysticercosis: a 23 years experience in the Hospital das Clínicas of Ribeirão Preto Medical School. Arq Neuropsiquiatr 1994;52(2): 166-186 